\title{
BIOPROCESSOS PARA REMOÇÃO DE DIÓXIDO DE CARBONO E ÓXIDO DE NITROGÊNIO POR MICRO- ALGAS VISANDO A UTILIZAÇÃ̃O DE GASES GERADOS DURANTE A COMBUSTÃO DO CARVÃO
}

\author{
Michele Greque de Morais e Jorge Alberto Vieira Costa* \\ Departamento de Química, Fundação Universidade Federal do Rio Grande, CP 474, 96201-900 Rio Grande - RS, Brasil
}

Recebido em 19/3/07; aceito em 11/10/07; publicado na web em 9/4/08

\begin{abstract}
BIOPROCESSES FOR REMOVAL OF CARBON DIOXIDE AND NITROGEN OXIDE BY MICROALGAE FOR THE UTILIZATION OF GAS GENERATED DURING COAL BURNING. The aim of this work was to study the removal of $\mathrm{CO}_{2}$ and NO by microalgae and to evaluate the kinetic characteristics of the cultures. Spirulina sp. showed $\mu_{\max }$ and $\mathrm{X}_{\max }\left(0.11 \mathrm{~d}^{-1}, 1.11 \mathrm{~g} \mathrm{~L}^{-}\right.$ ${ }^{1} \mathrm{~d}^{-1}$ ) when treated with $\mathrm{CO}_{2}$ and $\mathrm{NaNO}_{3}$. The maximum $\mathrm{CO}_{2}$ removal was $22.97 \%$ for $S$. obliquus treated with $\mathrm{KNO}_{3}$ and atmospheric $\mathrm{CO}_{2}$. The S. obliquus showed maximum $\mathrm{NO}$ removal $(21.30 \%)$ when treated with $\mathrm{NO}$ and $\mathrm{CO}_{2}$. Coupling the cultivation of these microalgae with the removal of $\mathrm{CO}_{2}$ and $\mathrm{NO}$ has the potential not only to reduce the costs of culture media but also to offset carbon and nitrogen emissions.
\end{abstract}

Keywords: air pollution; biofixation; microalgae.

\section{INTRODUÇÃO}

O carvão mineral é o mais abundante dos combustíveis fósseis, com reservas mundiais estimadas em 1 trilhão de $\mathrm{t}$, o suficiente para atender à demanda atual por mais de 200 anos. No Brasil, as principais reservas de carvão mineral estão localizadas no Sul do País, em especial no Rio Grande do Sul, que detém mais de $90 \%$ das reservas nacionais. No final de 2002, as reservas nacionais de carvão estavam em torno de 12 bilhões de t, o que corresponde a mais de $50 \%$ das reservas sul-americanas e a $1,2 \%$ das reservas mundiais. ${ }^{1}$

No Rio Grande do Sul a queima de combustíveis fósseis, principalmente carvão, está entre as fontes industriais que têm provocado alterações da qualidade ambiental em determinadas áreas, como ocorre na região de Candiota, localizada ao sudoeste do Estado. No entanto, a viabilidade da utilização do carvão está vinculada à redução das emissões de gases originados de sua queima, como o dióxido de carbono $\left(\mathrm{CO}_{2}\right)$ e os óxidos de enxofre $\left(\mathrm{SO}_{x}\right)$ e nitrogênio $\left(\mathrm{NO}_{x}\right)$.

Os níveis de $\mathrm{CO}_{2}$ na atmosfera aumentaram de 260 a 380 ppm nos últimos 100 anos, ${ }^{2}$ principalmente devido à queima de combustíveis fósseis associada ao aumento da população e industrialização. ${ }^{3}$ As alterações climáticas geradas pela emissão de gases responsáveis pelo efeito estufa, como o $\mathrm{CO}_{2}$, podem causar impactos desastrosos, como a redução da produção agrícola, alteração no suprimento de água doce, extinção de espécies, tempestades e enchentes.

As emissões de $\mathrm{NO}_{\mathrm{x}}$ no mundo são de 10 milhões de t por ano, provenientes de fontes naturais e 40 milhões de $t$ por ano de fontes antropogênicas oriundas principalmente dos processos de combustão. ${ }^{4} \mathrm{O}$ óxido de nitrogênio (NO) é o maior constituinte do $\mathrm{NO}_{\mathrm{x}} \mathrm{em}$ gases de combustão. O NO emitido na atmosfera é lentamente oxidado a $\mathrm{NO}_{2}$ no ar, resultando na chuva ácida. A quebra do equilíbrio do ecossistema natural causa acidificação do solo e da água e, conseqüentemente, alterações na biodiversividade. ${ }^{5}$

Globalmente, poucas alternativas viáveis têm sido apresentadas para redução da emissão de $\mathrm{CO}_{2}$ por parte das termelétricas, passando pela injeção deste gás no mar, ou em minas de carvão e poços de petróleo desativados. As pesquisas para redução de emis-

*e-mail: jorgealbertovc@ terra.com.br sões de NO têm sido conduzidas no sentido de substituir os processos de combustão atualmente utilizados, como a combustão em leito fluidizado, gaseificação integrada a ciclos combinados e uso de turbina a gás. ${ }^{1}$

Os microrganismos fotossintéticos, em especial as microalgas, têm sido utilizados no seqüestro e assimilação do dióxido de carbono. As fontes de nutrientes como carbono e nitrogênio no cultivo de microalgas representam importantes componentes, de modo que reduzir a quantidade de meio inorgânico, sem perder em produtividade, pode ser um artifício para minimizar custos de produção. ${ }^{6} \mathrm{~A}$ remoção de $\mathrm{CO}_{2}$ e $\mathrm{NO}$ por microalgas pode ser um dos mais eficientes processos de redução desses gases, sem a necessidade de mudanças radicais na matriz energética mundial e nas atividades produtivas.

O objetivo deste trabalho foi verificar a capacidade de remoção de dióxido de carbono e óxido de nitrogênio por microalgas, bem como avaliar as características cinéticas dos cultivos.

\section{PARTE EXPERIMENTAL}

\section{Microrganismos e meios de cultivo}

Os microrganismos utilizados foram as microalgas Spirulina sp. cepa LEB 18 e Scenedesmus obliquus cepa LEB 22.7 O meio utilizado para preparação e manutenção do inóculo de Spirulina sp. utilizado nos ensaios sem $\mathrm{NO}$ e $\mathrm{CO}_{2}$ foi meio Zarrouk original, ${ }^{8}$ contendo $\left(\mathrm{g} \mathrm{L}^{-1}\right)$ : $\mathrm{NaHCO}_{3}, 16,8 ; \mathrm{NaNO}_{3}, 2,5 ; \mathrm{K}_{2} \mathrm{HPO}_{4}, 0,5 ; \mathrm{K}_{2} \mathrm{SO}_{4}$, 1,$0 ; \mathrm{NaCl}, 1,0 ; \mathrm{Mg}_{.} \mathrm{SO}_{4} .7 \mathrm{H}_{2} \mathrm{O}, 0,2 ; \mathrm{CaCl}_{2}, 0,04 ; \mathrm{FeSO}_{4} \cdot 7 \mathrm{H}_{2} \mathrm{O}, 0,01$; EDTA, 0,08 e micronutrientes. Nos ensaios com adição de $\mathrm{NO}$ e $\mathrm{CO}_{2} \mathrm{o}$ inóculo foi aclimatado por 7 dias em ar misturado a $\mathrm{NO}$ e $\mathrm{CO}_{2}$ em Zarrouk modificado ${ }^{9}$ sem fonte original de nitrogênio $\left(\mathrm{NaNO}_{3}\right)$ e carbono $\left(\mathrm{NaHCO}_{3}\right)$.

Para preparação e manutenção do inóculo da microalga Scenedesmus obliquus utilizado nos ensaios sem adição de $\mathrm{NO}$ e $\mathrm{CO}_{2}$ utilizou-se meio $\mathrm{MC}$ original, ${ }^{10}$ contendo $\left(\mathrm{g} \mathrm{L}^{-1}\right)$ : $\mathrm{KNO}_{3}, 1,25$; $\mathrm{MgSO}_{4} .7 \mathrm{H}_{2} \mathrm{O}, 1,25 ; \mathrm{KH}_{2} \mathrm{PO}_{4}, 1,25 ; \mathrm{FeSO}_{4} .7 \mathrm{H}_{2} \mathrm{O}, 0,02$ e micronutrientes. Nos ensaios com adição de $\mathrm{NO}$ e $\mathrm{CO}_{2} \mathrm{O}$ inóculo foi aclimatado por 7 dias em ar misturado a $\mathrm{NO}$ e $\mathrm{CO}_{2}$ em MC modificado sem a fonte original de nitrogênio $\left(\mathrm{KNO}_{3}\right)$. 


\section{Condições de cultivo e planejamento experimental}

As microalgas foram cultivadas em fotobiorreatores do tipo erlenmeyer de 2,0 L com volume útil de 1,8 L. A aeração foi realizada misturando ar comprimido ao $\mathrm{CO}_{2}$ e $\mathrm{NO}$ através de um cilindro industrial (White Martins - Brasil) com vazão de 0,3 vvm, contendo $6 \%$ de $\mathrm{CO}_{2}(\mathrm{v} / \mathrm{v})$ e $100 \mathrm{ppm}$ de $\mathrm{NO}(\mathrm{v} / \mathrm{v})$. Os gases foram adicionados aos cultivos por $15 \mathrm{~min}$, a cada $2 \mathrm{~h}$, durante o período claro. ${ }^{9}$ A iluminância foi 3200 Lux fornecida por lâmpadas fluorescentes tipo luz do dia (General Eletric, $40 \mathrm{~W}$ ). Os cultivos com duração de 20 dias tiveram concentração inicial de biomassa 0,15 $\mathrm{g} \mathrm{L}^{-1}$. O aparato experimental foi mantido em câmara termostatizada a $35{ }^{\circ} \mathrm{C}$ com ciclos de claro/escuro alternando-se a cada 12 h. ${ }^{11}$

Para avaliação dos cultivos foi aplicado um planejamento fatorial completo $2^{2}$ para cada microalga em duplicata ${ }^{12}$ (Tabela 1). As variáveis estudadas foram a fonte de carbono $\left(\mathrm{X}_{1}\right.$; dióxido de carbono e fonte de carbono do meio de cultivo original de cada microalga) e a fonte de nitrogênio $\left(X_{2}\right.$; óxido de nitrogênio e fonte de nitrogênio do meio de cultivo original de cada microalga) e as respostas avaliadas foram as velocidades específicas máximas de crescimento $\left(\mu_{\max }, \mathrm{d}^{-1}\right)$, concentração celular máxima $\left(\mathrm{X}_{\max }, \mathrm{g} \mathrm{L}^{-1}\right)$, produtividades máximas $\left(\mathrm{P}_{\max }, \mathrm{g} \mathrm{L}^{-1} \mathrm{~d}^{-1}\right)$, acúmulo de carbono removido (RAC, g), remoção média de carbono ( $\mathrm{RC}, \mathrm{g} \mathrm{g}^{-1}$ ), acúmulo de nitrogênio removido (RAN, g) e remoção média de nitrogênio (RN, $\left.\mathrm{g} \mathrm{g}^{-1}\right)$.

Tabela 1. Níveis reais e codificados das variáveis estudadas no planejamento fatorial completo $2^{2}$ para Spirulina sp. e Scenedesmus obliquus

\begin{tabular}{|c|c|c|c|c|}
\hline \multicolumn{5}{|c|}{ Spirulina sp. } \\
\hline 1 & $\mathrm{NaHCO}_{3}$ & $\mathrm{NaNO}_{3}$ & -1 & -1 \\
\hline 2 & $6 \% \mathrm{CO}_{2}$ & $\mathrm{NaNO}_{3}$ & +1 & -1 \\
\hline 3 & $\mathrm{NaHCO}_{3}$ & NO & -1 & +1 \\
\hline 4 & $6 \% \mathrm{CO}_{2}$ & NO & +1 & +1 \\
\hline \multicolumn{5}{|c|}{ Scenedesmus obliquus } \\
\hline 5 & $0,038 \% \mathrm{CO}_{2}$ & $\mathrm{KNO}_{3}$ & -1 & -1 \\
\hline 6 & $6 \% \mathrm{CO}_{2}$ & $\mathrm{KNO}_{3}$ & +1 & -1 \\
\hline 7 & $0,038 \% \mathrm{CO}_{2}$ & $\mathrm{NO}$ & -1 & +1 \\
\hline 8 & $6 \% \mathrm{CO}_{2}$ & NO & +1 & +1 \\
\hline
\end{tabular}

$\mathrm{NaHCO}_{3}: 16,8 \mathrm{~g} \mathrm{~L}^{-1}$ original do meio de cultivo; $\mathrm{NaNO}_{3}: 2,5 \mathrm{~g} \mathrm{~L}^{-1}$ original do meio de cultivo; $0,038 \%$ de $\mathrm{CO}_{2}$ : original do ar atmosférico; $\mathrm{KNO}_{3}: 1,25 \mathrm{~g} \mathrm{~L}^{-1}$ original do meio de cultivo; $\mathrm{X}_{1}$ : fonte de carbono; $\mathrm{X}_{2}$ : fonte de nitrogênio.

\section{Respostas estudadas}

Os valores da concentração celular foram usados para calcular as velocidades específicas máximas de crescimento $\left(\mu_{\max }, \mathrm{d}^{-1}\right)$, concentração celular máxima $\left(\mathrm{X}_{\max }, \mathrm{g} \mathrm{L}^{-1}\right)$ e produtividades máximas $\left(\mathrm{P}_{\max }, \mathrm{g} \mathrm{L}^{-1} \mathrm{~d}^{-1}\right)$. A velocidade específica máxima de crescimento $\left(\mu_{\max }, d^{-1}\right)$ foi calculada pela regressão exponencial da fase logarítmica da curva de crescimento celular. A concentração celular máxima é o máximo valor de concentração obtido. A produtividade $\left(\mathrm{P}, \mathrm{g} \mathrm{L}^{-1} \mathrm{~d}^{-1}\right)$ foi obtida segundo a equação $\mathrm{P}=\left(\mathrm{X}_{\mathrm{t}}-\mathrm{X}_{\mathrm{o}}\right)$. $\left(\mathrm{t}-\mathrm{t}_{\mathrm{o}}\right)^{-1}$, onde $\mathrm{X}_{\mathrm{t}}$ é a concentração celular $\left(\mathrm{g} \mathrm{L}^{-1}\right)$ no tempo $\mathrm{t}(\mathrm{d})$ e $\mathrm{X}_{\mathrm{o}}$ $\left(\mathrm{g} \mathrm{L}^{-1}\right)$ a concentração celular no tempo $\mathrm{t}_{\mathrm{o}}(\mathrm{d}) .{ }^{13} \mathrm{P}_{\max }$ é o máximo valor de produtividade encontrado ao longo do cultivo. ${ }^{14}$

Para os cálculos de remoção de carbono utilizaram-se os valores de concentração de carbono na biomassa de cada microalga obtidos na análise de CHNS. O acúmulo de carbono removido (RAC, g) foi determinado segundo a equação $\mathrm{RAC}=\left(\mathrm{X}_{\mathrm{t}}-\mathrm{X}_{\mathrm{o}}\right)$ . $\mathrm{m}_{\mathrm{CBM}} \cdot \mathrm{V}_{\mathrm{FBR}} \cdot\left(\mathrm{m}_{\mathrm{FCU}} \cdot \mathrm{m}_{\mathrm{C}}{ }^{-1}\right)$, onde $\mathrm{X}_{\mathrm{t}}\left(\mathrm{g} \mathrm{L}^{-1}\right)$ é a concentração celular no tempo $t(\mathrm{~d}), \mathrm{X}_{\mathrm{o}}\left(\mathrm{g} \mathrm{L}^{-1}\right)$ a concentração celular no tempo $\mathrm{t}_{\mathrm{o}}, \mathrm{m}_{\mathrm{CBM}}(\mathrm{g}$ $\mathrm{g}^{-1}$ ) é a fração mássica de carbono determinada na biomassa microalgal, $\mathrm{V}_{\mathrm{FBR}}(\mathrm{L})$ é o volume de meio no fotobiorreator, $\mathrm{m}_{\mathrm{FCU}}(\mathrm{g}$ $\left.\mathrm{mol}^{-1}\right)$ é a massa molar da fonte de carbono utilizada e $\mathrm{m}_{\mathrm{C}}\left(\mathrm{g} \mathrm{mol}^{-1}\right)$ é a massa molar do carbono.

A remoção média de carbono $\left(\mathrm{RC}, \mathrm{g} \mathrm{g}^{-1}\right)$ foi determinada pela equação $\mathrm{RC}=\left(\mathrm{RACl}_{(\mathrm{t}+1)}-\mathrm{RAC1} 1_{1}\right) \cdot\left[\mathrm{m}_{\mathrm{cid}} \cdot(\mathrm{t}+1)^{-1}\right]^{-1}$, onde $\mathrm{RAC1} 1_{(\mathrm{t}+1)}$ é o acúmulo de carbono removido no tempo t+1 (d). RAC1 1 é o acúmulo de carbono no tempo $\mathrm{t}=1 \mathrm{~d}, \mathrm{~m}_{\text {cid }}$ ( $\mathrm{g}$ ) é a massa de carbono injetada diariamente no tempo $\mathrm{t}+1$ (d).

Para os cálculos de remoção de nitrogênio utilizaram-se os valores de concentração de nitrogênio na biomassa de cada microalga, obtidos através de análise de CHNS. O acúmulo de nitrogênio removido (RAN, g) foi determinado segundo a equação $\mathrm{RAN}=\left(\mathrm{X}_{\mathrm{t}}-\mathrm{X}_{\mathrm{o}}\right) \cdot \mathrm{m}_{\mathrm{NBM}} \cdot \mathrm{V}_{\mathrm{FBR}} \cdot\left(\mathrm{m}_{\mathrm{FNU}} \cdot \mathrm{m}_{\mathrm{N}}^{-1}\right)$, onde $\mathrm{X}_{\mathrm{t}}\left(\mathrm{g} \mathrm{L}^{-1}\right)$ é a concentração celular no tempo $t(d), X_{o}\left(g^{-1}\right)$ a concentração celular no tempo $\mathrm{t}_{\mathrm{o}}, \mathrm{m}_{\mathrm{NBM}}\left(\mathrm{g} \mathrm{g}^{-1}\right)$ é a fração mássica de nitrogênio determinada na biomassa microalgal, $\mathrm{V}_{\mathrm{FBR}}(\mathrm{L})$ é o volume de meio no fotobiorreator, $\mathrm{m}_{\mathrm{FNU}}\left(\mathrm{g} \mathrm{mol}^{-1}\right)$ é a massa molar da fonte de nitrogênio utilizada e $\mathrm{m}_{\mathrm{N}}\left(\mathrm{g} \mathrm{mol}^{-1}\right)$ é a massa molar do nitrogênio.

A remoção média de nitrogênio $\left(\mathrm{RN}, \mathrm{g} \mathrm{g}^{-1}\right)$ foi determinada pela equação $R N=\left(R A N 1_{(t+1)}-R A N 1_{1}\right) \cdot\left[m_{\text {nid }} \cdot(t+1)^{-1}\right]^{-1}$, onde $\mathrm{RAN1}_{(\mathrm{t}+1)}$ é o acúmulo de nitrogênio removido no tempo $\mathrm{t}+1$ (d). $\mathrm{RAN}_{1}$ é o acúmulo de nitrogênio no tempo $\mathrm{t}=1 \mathrm{~d}, \mathrm{~m}_{\text {nid }}(\mathrm{g})$ é a massa de nitrogênio injetada diariamente no tempo $t+1$ (d).

\section{Determinações analíticas}

A cada 24 h coletaram-se amostras assepticamente para acompanhamento da concentração celular, calculada através da medida da densidade ótica a $670 \mathrm{~nm}^{11}$ em espectrofotômetro (Femto 700 Plus, Brasil), que foi comparada com curva de calibração relacionando densidade ótica e peso seco de biomassa para cada microalga. ${ }^{11}$

Determinações de carbono e nitrogênio foram realizadas em analisador elementar CHNS (Perkin Elmer 2400) e os resultados foram utilizados para os cálculos de remoção de carbono e nitrogênio. A calibração do equipamento foi realizada utilizando o padrão de cistina certificado (Perkin Elmer, USA).

\section{Análise estatística}

Os resultados obtidos a partir do planejamento experimental fatorial completo $2^{2}$ para cada microalga foram avaliados através de Análise de Variância (ANOVA) e Efeitos de Interação. Também foi realizado teste de Tukey para comparação de médias dos parâmetros cinéticos, remoção de $\mathrm{CO}_{2}$ e $\mathrm{NO}$ das duplicatas dos ensaios com nível de significância de $90 \%$ (p $\leq 0,10) .{ }^{12}$

\section{RESULTADOS E DISCUSSÃO}

A Tabela 2 e Figuras 1, 2 e 3 apresentam as respostas cinéticas e de remoção de carbono e nitrogênio dos cultivos realizados.

As microalgas Spirulina sp. e S. obliquus não apresentaram diferença significativa quanto à velocidade específica máxima de crescimento. Os valores superiores de velocidade específica máxima de crescimento alcançado pelas microalgas Spirulina sp. e $S$. obliquus foram 0,11 e 0,10 $\mathrm{d}^{-1}$, respectivamente. Segundo Morais e Costa, ${ }^{9}$ a microalga Spirulina sp. cultivada com $6 \%$ de $\mathrm{CO}_{2}$ em fotobiorreator tubular vertical obteve velocidade específica máxima de crescimento de $0,18 \mathrm{~d}^{-1}$. 
Tabela 2. Concentração celular máxima $\left(\mathrm{X}_{\max }, \mathrm{g} \mathrm{L}^{-1}\right)$, produtividade máxima $\left(\mathrm{P}_{\max }, \mathrm{g} \mathrm{L}^{-1} \mathrm{~d}^{-1}\right)$, velocidade específica máxima de crescimento $\left(\mu_{\max }, \mathrm{d}^{-1}\right)$, remoção média de carbono $(\mathrm{RC}, \%)$ e remoção média de nitrogênio (RN, \%) para Spirulina sp. e Scenedesmus obliquus cultivadas durante $20 \mathrm{~d}$ segundo o planejamento fatorial completo $2^{2}$

\begin{tabular}{lccccc}
\hline Ensaio & $\mathrm{X}_{\max }$ & $\mathrm{P}_{\max }$ & $\mu_{\max }$ & $\mathrm{RC}$ & $\mathrm{RN}$ \\
& & & Spirulina $\mathrm{sp.}$ & \\
1 & $1,00 \pm 0,14$ & $0,05 \pm 0,05$ & $0,08 \pm 0,01$ & $9,54 \pm 1,53$ & $14,00 \pm 2,19$ \\
2 & $1,11 \pm 0,14$ & $0,06 \pm 0,01$ & $0,11 \pm 0,02$ & $2,67 \pm 0,51$ & $23,40 \pm 3,76$ \\
3 & $0,96 \pm 0,13$ & $0,05 \pm 0,00$ & $0,08 \pm 0,01$ & $10,55 \pm 1,76$ & $15,93 \pm 4,21$ \\
4 & $1,02 \pm 0,17$ & $0,05 \pm 0,01$ & $0,09 \pm 0,03$ & $2,10 \pm 0,99$ & $17,55 \pm 0,82$ \\
\hline 5 & & Scenedesmus obliquus & & $46,12 \pm 2,07$ \\
6 & $0,86 \pm 0,06$ & $0,06 \pm 0,05$ & $0,09 \pm 0,00$ & $22,97 \pm 9,59$ & $45,48 \pm 3,42$ \\
7 & $0,86 \pm 0,05$ & $0,06 \pm 0,00$ & $0,10 \pm 0,01$ & $3,85 \pm 0,01$ & $13,88 \pm 1,14$ \\
\hline & $0,70 \pm 0,03$ & $0,03 \pm 0,00$ & $0,08 \pm 0,01$ & $18,42 \pm 4,39$ & $27,13 \pm 1,72$ \\
\hline
\end{tabular}

Os valores apresentados representam a média \pm desvio padrão.

Com exceção da concentração celular máxima dos ensaios de Spirulina sp. cultivada com $6 \% \mathrm{CO}_{2}$ e $\mathrm{NaNO}_{3}\left(1,11 \mathrm{~g} \mathrm{~L}^{-1}\right)$ e $S$. obliquus cultivada com $0,038 \%$ de $\mathrm{CO}_{2}$ e $\mathrm{NO}\left(0,70 \mathrm{~g} \mathrm{~L}^{-1}\right)$, os parâmetros cinéticos analisados não apresentaram diferenças significativas $(\mathrm{p}>0,25)$, independente da fonte de carbono e nitrogênio utilizadas, mostrando o potencial de remoção de dióxido de carbono e óxido de nitrogênio, sem prejudicar o crescimento microalgal.

Nos cultivos realizados com a microalga $S$. obliquus observouse efeito de interação significativo $(\mathrm{p}=0,03)$ entre a fonte de carbono e nitrogênio, mostrando que a concentração celular foi máxima $\left(0,98 \pm 0,10 \mathrm{~g} \mathrm{~L}^{-1}\right)$ quando foi utilizado $\mathrm{NO}$ e $6 \% \mathrm{CO}_{2}$ combinados. A microalga Spirulina sp. cultivada com $\mathrm{CO}_{2}$ e fonte de nitrogênio original do meio de cultivo $\left(\mathrm{NaNO}_{3}\right)$ foi a condição que apresentou máxima produtividade $\left(0,06 \mathrm{~g} \mathrm{~L}^{-1} \mathrm{~d}^{-1}\right)$.

A microalga Spirulina sp. cultivada com $\mathrm{CO}_{2}$ e $\mathrm{NaNO}_{3}$ apresentou crescimento superior aos demais ensaios (Figura 1), entretanto as curvas de crescimento para as diferentes condições foram estatisticamente iguais $(\mathrm{p}>0,10)$. A microalga $S$. obliquus não apresentou diferença significativa $(\mathrm{p}<0,02)$ entre as curvas de crescimento até o $5^{\circ}$ dia de cultivo. No $10^{\circ}$ dia a $S$. obliquus cultivada com $0,038 \%$ de $\mathrm{CO}_{2}$ e $\mathrm{NO}$ apresentou crescimento menor $(\mathrm{p}<0,04)$ que os ensaios com adição de $6 \%$ de $\mathrm{CO}_{2}$ e $\mathrm{KNO}_{3}$, e com adição de $6 \% \mathrm{CO}_{2}$ e NO. No $15^{\circ}$ dia de cultivo a S. obliquus apresentou diferença significativa $(\mathrm{p}<0,02)$ entre o ensaio utilizando fonte de carbono e nitrogênio original do meio e o ensaio utilizando $6 \%$ $\mathrm{CO}_{2}$ e NO.

A microalga Spirulina sp. a partir do $2^{\circ}$ dia de cultivo obteve maior taxa de remoção de carbono $(\mathrm{p}<0,02)$ no cultivo realizado com fontes de carbono e nitrogênio originais do meio, bem como aquele usando fonte de carbono original e NO. A S. obliquus apresentou o mesmo comportamento, entretanto a partir do $10^{\circ}$ dia de cultivo.

A remoção de carbono foi $2,67 \pm 0,51$ e $3,85 \pm 0,01 \%$, respectivamente, para as microalgas Spirulina sp. e S. obliquus nos ensaios realizados com $6 \% \mathrm{CO}_{2}$ e fonte de nitrogênio original do meio de cultivo $\left(\mathrm{NaNO}_{3}\right.$ e $\mathrm{KNO}_{3}$ ). Nos cultivos com $\mathrm{CO}_{2}$ e $\mathrm{NO}$ a microalga Spirulina sp. obteve 2,10 \pm 0,99\% de remoção de carbono e a $S$. obliquus alcançou $3,33 \pm 0,21$. A $S$. obliquus alcançou máxima remoção de carbono $(22,97 \%)$ no ensaio contendo fonte de carbono original do meio de cultivo $\left(0,038 \% \mathrm{CO}_{2}\right)$ e fonte de nitrogênio original do meio de cultivo $\left(\mathrm{KNO}_{3}\right)$.

$\mathrm{O}$ bicarbonato de sódio é o nutriente adicionado em maior quantidade no meio de cultivo original para Spirulina $\left(16,80 \mathrm{~g} \mathrm{~L}^{-1}\right)$, correspondendo a $60 \%$ do custo total com nutrientes, ${ }^{15}$ podendo a capacidade das microalgas em fixar $\mathrm{CO}_{2}$ ser usada para reduzir custos com nutrientes e os diversos problemas ambientais causados por esse gás.

Durante os 4 primeiros dias de cultivo a Spirulina sp. apresentou maior taxa de remoção de nitrogênio $(\mathrm{p}<0,01)$ nos ensaios com $\mathrm{NaHCO}_{3}$ e $\mathrm{NaNO}_{3}$, e utilizando $\mathrm{CO}_{2}$ e $\mathrm{NaNO}_{3}$. No $5^{\circ}$ dia de cultivo o ensaio com adição de $\mathrm{CO}_{2}$ e $\mathrm{NO}$ apresentou taxas de remoção de nitrogênio maior $(\mathrm{p}<0,02)$ que o cultivo utilizando
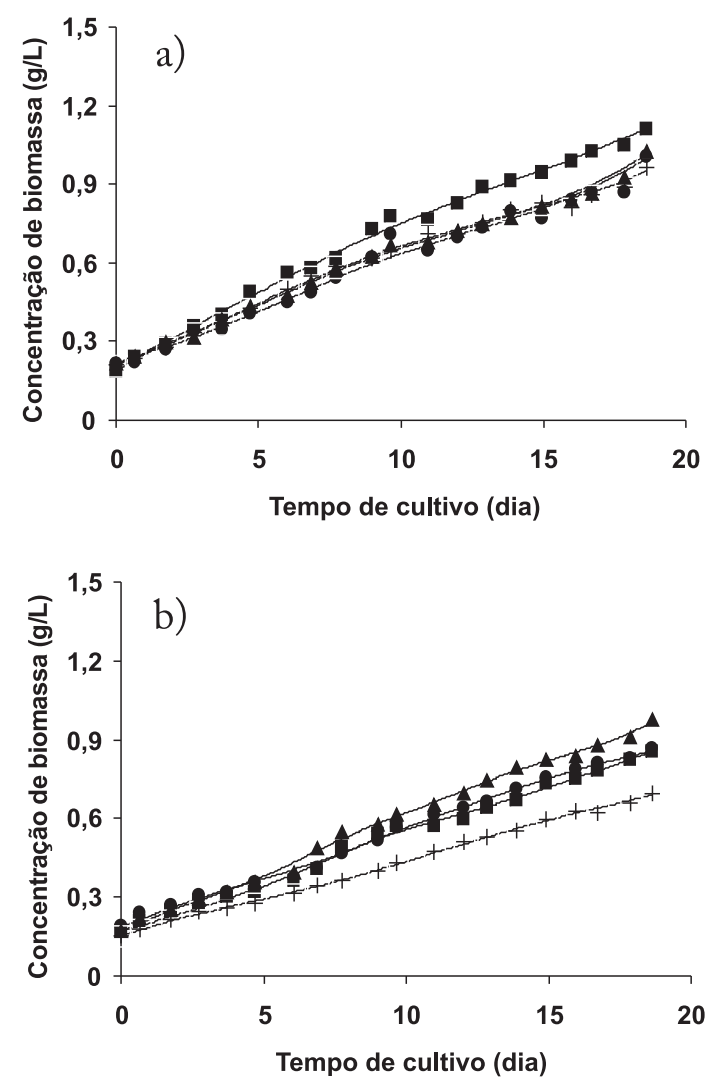

Figura 1. Concentração de biomassa em função do tempo para Spirulina sp. (a) e S. obliquus (b) cultivadas com adição de fonte de carbono e nitrogênio original do meio de cultivo $(\bullet), \mathrm{CO}_{2}$ como fonte de carbono e fonte de nitrogênio original do meio de cultivo (অ), fonte de carbono original do meio de cultivo e $\mathrm{NO}$ como fonte de nitrogênio $(+)$ e $\mathrm{CO}_{2}$ como fonte de carbono e NO como fonte de nitrogênio $(\mathbf{\Delta})$ 


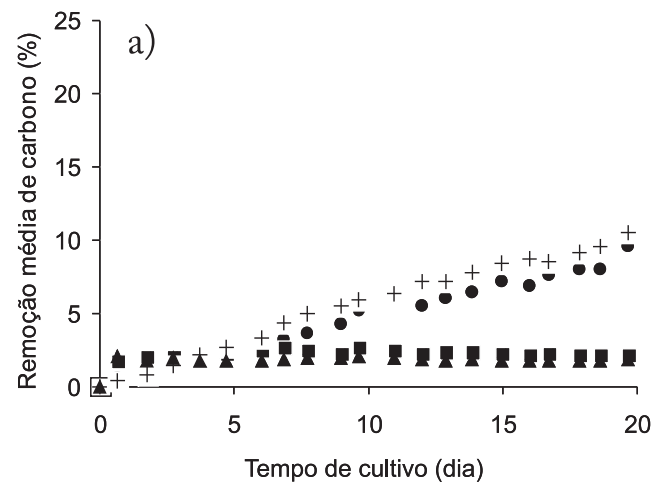

b)

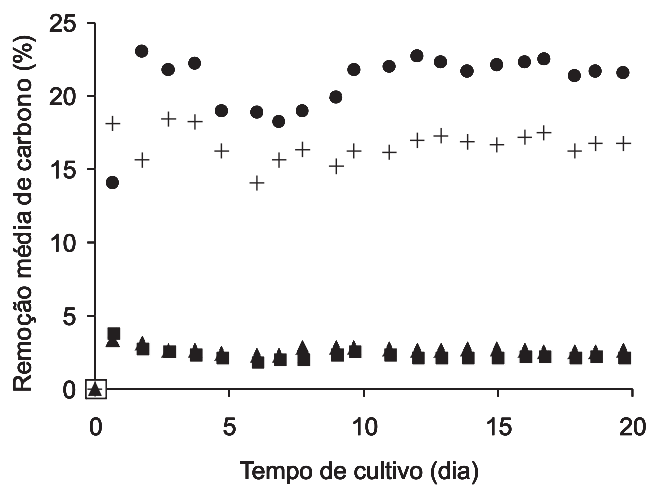

Figura 2. Remoção média de carbono para Spirulina sp. (a) e S. obliquus (b) cultivadas com adição de fonte de carbono e nitrogênio original do meio de cultivo $(\bullet), \mathrm{CO}_{2}$ como fonte de carbono e fonte de nitrogênio original do meio de cultivo ( $\mathbf{\square})$, fonte de carbono original do meio de cultivo e NO como fonte de nitrogênio (+) e $\mathrm{CO}_{2}$ como fonte de carbono e $\mathrm{NO}$ como fonte de nitrogênio ( $\mathbf{\Delta})$

$\mathrm{NaHCO}_{3}$ e $\mathrm{NaNO}_{3}$. A partir do $12^{\circ}$ dia de cultivo os ensaios não apresentaram diferença significativa $(\mathrm{p}>0,63)$ quanto à taxa de remoção de nitrogênio para a microalga Spirulina sp.

A máxima taxa de remoção de nitrogênio para a microalga Spirulina sp. foi $23,40 \%$ no ensaio com $\mathrm{CO}_{2}$ como fonte de carbono e fonte de nitrogênio original do meio $\left(\mathrm{NaNO}_{3}\right)$. A substituição da fonte de carbono original do meio de cultivo da microalga Spirulina sp. por $6 \% \mathrm{CO}_{2}$ proporcionou incremento de $6,40 \%$ na remoção de nitrogênio. $O$ efeito de interação significativo $(p=0,05)$ entre a fonte de nitrogênio e carbono mostrou que, independente da fonte de nitrogênio utilizada, os cultivos com a microalga Spirulina sp. apresentaram maior remoção de nitrogênio quando se utilizou $\mathrm{CO}_{2}$ como fonte de carbono. $\mathrm{O}$ ensaio com $\mathrm{CO}_{2}$ como fonte de carbono e $\mathrm{NO}$ como fonte de nitrogênio manteve a remoção média de nitrogênio estável no decorrer do ensaio, alcançando máximo de $17,55 \%$ com 20 dias de cultivo.

A $S$. obliquus aumentou a taxa de remoção de nitrogênio ao longo do tempo nos ensaios com fonte de carbono $\left(0,038 \%\right.$ de $\left.\mathrm{CO}_{2}\right)$ e nitrogênio $\left(\mathrm{KNO}_{3}\right)$ originais do meio $(46,12 \%)$ e nos cultivos realizados com $6 \% \mathrm{CO}_{2}$ e fonte de nitrogênio $\left(\mathrm{KNO}_{3}\right)$ original do meio $(45,48 \%)$. Até o $5^{\circ}$ dia de cultivo a microalga $S$. obliquus com adição de $\mathrm{CO}_{2}$ e $\mathrm{NO}$ obteve maior taxa de remoção de nitrogênio $(\mathrm{p}<0,02)$. A partir do $10^{\circ}$ dia, o cultivo com adição de fonte de carbono original do meio e NO apresentou a menor taxa de remoção de nitrogênio $(\mathrm{p}<0,01)$. Durante os 20 dias de ensaio, os cultivos com fontes de carbono e nitrogênio originais do meio e aqueles com $6 \% \mathrm{CO}_{2}$ e fonte de nitrogênio original do meio não apresentaram diferença significativa quanto à taxa de remoção de ni-
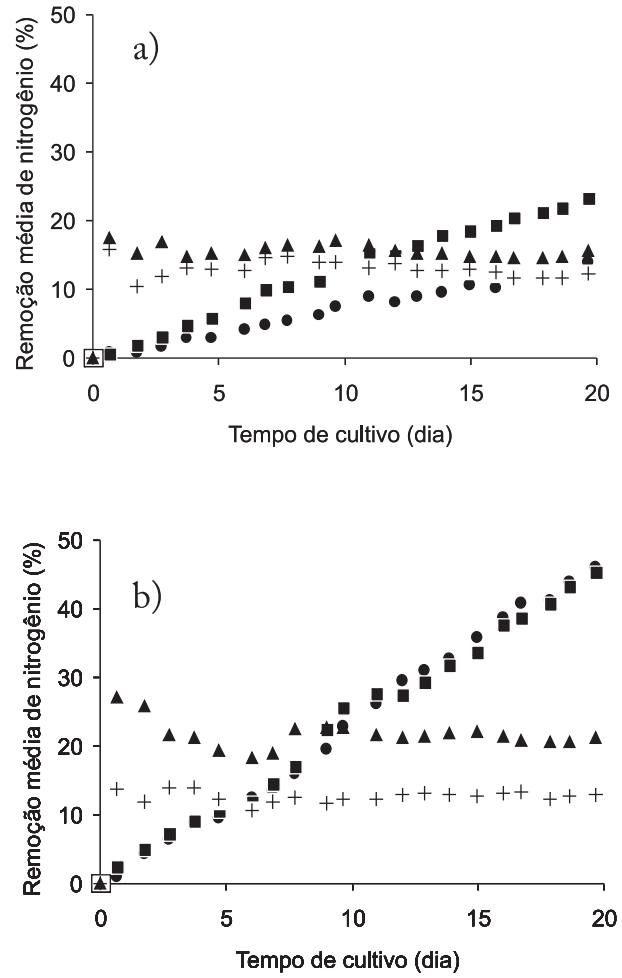

Figura 3. Remoção média de nitrogênio para Spirulina sp. (a) e S. obliquus (b) cultivadas com adição de fonte de carbono e nitrogênio original do meio de cultivo (•), $\mathrm{CO}_{2}$ como fonte de carbono e fonte de nitrogênio original do meio de cultivo (ם), fonte de carbono original do meio de cultivo e NO como fonte de nitrogênio (+) e $\mathrm{CO}_{2}$ como fonte de carbono e $\mathrm{NO}$ como fonte de nitrogênio ( $\mathbf{\Delta})$

trogênio $(\mathrm{p}>0,90)$.

Os ensaios com NO apresentaram remoção de nitrogênio de $27,13 \%$ utilizando $\mathrm{CO}_{2}$ como fonte de carbono e $13,88 \%$ com a fonte de carbono original do meio $\left(0,038 \%\right.$ de $\left.\mathrm{CO}_{2}\right)$. Segundo Nagase et al., ${ }^{16}$ o NO na fase gasosa é dissolvido no meio de cultivo e retirado pelas microalgas através de difusão, e assim o NO removido é preferencialmente utilizado como fonte de nitrogênio para o crescimento celular.

Nos ensaios realizados verificou-se que além das microalgas utilizarem NO, a adição de 100 ppm deste gás não prejudicou seu desenvolvimento. Yanagi et al. ${ }^{17}$ verificaram que a microalga Chlorella sp. foi tolerante a $120 \mathrm{ppm}$ de $\mathrm{NO}_{x}$, e a adição de 150 ppm deste gás não causou influência no crescimento celular de Chlorella sp. ${ }^{18}$ nem de Monoraphidium minutum. ${ }^{19}$

\section{CONCLUSÕES}

As maiores taxas de remoção de $\mathrm{CO}_{2}$ e $\mathrm{NO}$ foram obtidas com a microalga $S$. obliquus. A máxima remoção de $\mathrm{CO}_{2}$ foi $22,97 \%$ quando cultivada com $\mathrm{KNO}_{3}$ e $\mathrm{CO}_{2}$ atmosférico e a máxima remoção de $\mathrm{NO}$ foi $27,13 \%$, quando cultivada com $\mathrm{NO}$ e $6 \% \mathrm{CO}_{2}$.

A microalga Spirulina sp. quando cultivada com $\mathrm{CO}_{2}$ e $\mathrm{NaNO}_{3}$ obteve os máximos valores para velocidade específica $\left(0,11 \mathrm{~d}^{-1}\right) \mathrm{e}$ concentração celular $\left(1,11 \mathrm{~g} \mathrm{~L}^{-1} \mathrm{~d}^{-1}\right)$. A $S$. obliquus alcançou máxima concentração celular $\left(0,98 \mathrm{~g} \mathrm{~L}^{-1}\right)$ quando cultivada com $\mathrm{CO}_{2} \mathrm{e}$ NO. A máxima velocidade específica alcançada pela microalga $S$. obliquus foi $0,10 \mathrm{~d}^{-1}$, quando cultivada com $\mathrm{CO}_{2}$, independente da fonte de nitrogênio utilizada. Aliar o cultivo de microalgas com a 
remoção de $\mathrm{CO}_{2}$ e $\mathrm{NO}$ pode reduzir os custos com nutrientes do meio de cultivo e problemas gerados com a emissão desses gases.

\section{AGRADECIMENTOS}

À ELETROBRAS - Centrais Elétricas Brasileiras S.A. e CGTEE - Companhia de Geração Térmica de Energia Elétrica pelo apoio financeiro para a realização desse trabalho.

\section{REFERÊNCIAS}

1. http://anell.gov.br/aplicacoes/atlas/pdf/08-carvao(2).pdf, acessada em Março 2007.

2. Siegenthaler, U.; Stocker, T. F.; Monnin, E.; Lüthi, D.; Schwander, J.; Stauffer, B.; Raynaud, D.; Barnola, J. M.; Fischer, H.; Delmontt, V. M.; Jouzel, J.; Science 2005, 310, 1313.

3. Chang, E. H.; Yang, S. S.; Bot. Bull. Acad. Sin. 2003, 44, 43.

4. Cónsul, J. M. D.; Thiele, D.; Veses, R. C.; Baibich, I. M.; Quim. Nova 2004, $27,432$.

5. Nagase, H.; Eguchi, K.; Yoshihara, K.; Hirata, K.; Miyamoto, K.; J. Ferment. Bioeng. 1998, 86, 421.

6. Beneman, J. R.; Hughes, E.; Energy Convers. Manage. 1997, 38, S467.
7. Morais, M. G.; Costa, J. A. V.; Energy Convers. Manage. 2007, 48, 2169

8. Zarrouk, C.; Tese de Doutorado, University of Paris, França, 1966.

9. Morais, M. G.; Costa, J. A. V.; J. Biotechnol. 2007, 129, 439.

10. Watanabe, A.; J. Gen. Appl. Microbiol. 1960, 6, 1.

11. Costa, J. A. V.; Morais, M. G.; Dalcanton, F.; Reichert, C. C.; Durante, A. J.; Z. Naturforsch., C: J. Biosci. 2006, 61, 105.

12. Box, G. E.; Hunter, W. G.; Hunter, J. S.; Statistics for experiments, John Willey \& Sons: USA, 1978

13. Schmidell, W.; Lima, A. U.; Aquarone, E.; Borzani, W.; Biotecnologia Industrial, Edgard Blücher Ltda.: São Paulo, 2001, vol. 2.

14. Bailey, J. E.; Ollis, D. F.; Biochemical Engineering Fundamentals, $2^{\text {nd }}$ ed., McGraw-Hill: Singapore, 1986.

15. Alava, D.; Mello, P. C.; Wagener, K.; J. Braz. Chem. Soc. 1997, 8, 447.

16. Nagase, H.; Eguchi, K.; Yoshihara, K.; Eguchi, K.; Okamoto, Y.; Murasaki, S.; Yamashita, R.; Hirata, K.; Miyamoto, K.; Biochem. Eng. J. 2001, 7, 241.

17. Yanagi, M.; Watanabe, Y.; Saiki, H.; Energy Convers. Manage. 1995, 36, 713.

18. Maeda, K.; Owada, M.; Kimura, N.; Omata, K.; Karube, I.; Energy Convers. Manage. 1995, 36, 717.

19. Zeiler, K. G.; Brown, L. M. Em Proceedings of the Air \& Waste Management Association Specialty Conference (Phoenix, AZ, April 5-8, 1994), Global Climate Change: Science, Policy and Mitigation Strategies,. Mathai, C. V.: G., Stensland, eds.; Air \& Waste Management Association: Pittsburgh, p. 947. 